\section{Review Article}

Korean J Transplant 2021;35:208-217 https://doi.org/10.4285/kjt.21.0033

\section{$\mathrm{KJT}^{<}$ OF TRANSPLANTATION}

pISSN 2671-8790

elSSN 2671-8804

\title{
Mycobacterial infections in solid organ transplant recipients
}

\author{
Harun Ur Rashid, Nura Afza Salma Begum, Tasnuva Sarah Kashem
}

Received December 22, 2021

Accepted December 25, 2021

Department of Nephrology, Kidney Foundation Hospital and Research Institute, Dhaka, Bangladesh

Corresponding author: Harun Ur Rashid Department of Nephrology, Kidney Foundation Hospital and Research Institute, Plot no. 5/2, Road no. I, section 2, Mirpur, Dhaka 1216, Bangladesh Tel: +880-18-1924-7299

E-mail: rashid@bol-online.com

\begin{abstract}
Mycobacterium tuberculosis (MTB) infection in solid organ transplant (SOT) recipients remains a major challenge for physicians and surgeons. Active tuberculosis (TB) is associated with increased morbidity and mortality in SOT recipients. MTB usually develops after transplantation in a recipient with latent TB infection (LTBI) before transplantation and may also be transmitted from the donor or acquired from the community. Therefore, screening for LTBI in donors and recipients before transplantation is very important in preventing active disease after transplantation. This review article is based on recently published data, case series, and expert recommendations. We reviewed updated information about the epidemiology, diagnosis, and treatment of latent and active TB before and after transplantation. We also reviewed recent treatments for multidrug-resistant TB.
\end{abstract}

Keywords: Mycobacterium tuberculosis; Latent tuberculosis infection; Solid organ transplantation; Multidrug-resistant tuberculosis

\section{INTRODUCTION}

Approximately $25 \%$ of the world's population is infected with Mycobacterium tuberculosis (MTB). The highest number of deaths among infectious diseases after coronavirus disease 2019 (COVID-19) was due to tuberculosis (TB). One of the major risk factors for active TB is living in lowand low-middle-income countries. Almost two-thirds of people infected with MTB are found in India, Bangladesh, China, Indonesia, Nigeria, Pakistan, the Philippines, and South Africa. There are several risk factors of TB, most important of which is the individual's immunological state [1]. MTB in transplant recipients is $20-70$ times higher than that in the general population [2]. Therefore, it is common for solid organ transplant (SOT) recipients to have MTB infection. Microbial-specific cytotoxic T cells, a key host defense against mycobacterial infection, are compromised by immunosuppressant drugs. The accurate diagnosis of latent TB infection (LTBI) and active TB in SOT patients becomes complicated due to its atypical presentations and negative smear tests for acid-fast Bacilli despite the presence of active TB, indeterminate tuberculin skin test (TST), and interferon gamma release assay (IGRA). The treatment of MTB in SOT patients is challenging, and the mortality rate of posttransplant TB cases is very high $[3,4]$.

\section{EPIDEMIOLOGY}

Dr. Robert Koch first identified MTB on March 24, 1882, which was commemorated as the World TB Day [5]. The global TB incidence is approximately $2 \%$ annually, and the cumulative reduction between 2015 and 2020 was $11 \%$. An estimated 66 million lives were saved through TB diagnosis and treatment between 2000 and 2020 [1]. 


\section{HIGHLIGHTS}

- Active tuberculosis (TB) is associated with increased morbidity and mortality in solid organ transplant recipients.

- Screening for latent TB infection in donor and recipient before transplant is very important in preventing active disease after transplantation.

- Management of TB is becoming difficult due to the emergence of multidrug-resistant cases.

Unfortunately, the COVID-19 pandemic has had a profound impact on global TB progress and TB-related expenditure. It is estimated that the COVID-19 pandemic will result in substantial additional TB burden in the next 5 years, adding US $\$ 1.95$ billion to TB-related health spending in India, \$29-43 million in Kenya, and \$45.38 million in Ukraine [6]. The number of organ transplants performed worldwide falls significantly during the first year of the COVID-19 pandemic [7].

MTB remains a major public health threat and challenge in patients with SOT. Data regarding the incidence and prevalence of MTB in SOT recipients are also limited. Among SOT patients, the incidence of active TB is estimated to be 20-74 times greater than that in the general population [3]. In developed countries, the prevalence of MTB in SOT recipients ranges from $1.2 \%$ to $6.4 \%$, whereas in developing countries in Asia and Africa, it ranges from $12 \%$ to $15 \%[3,8]$. The incidence of posttransplant TB was evaluated in a UK. study that included patients from different ethnic origins. This study found that the TB incidence rate was higher in the first year posttransplant and was highest among the Asian population (289.8/100,000 person-years in the Asian ethnic group, 30/100,000 person-years in the white ethnic group, and 33.3/100,000 person-years in the other ethnic group) [9]. Others also reported that MTB usually develops within the first year after transplantation, and the average time for presentation is usually $6-11$ months $[4,10]$. Mortality among posttransplant TB cases has been reported to be high at $20 \%-30 \%$ [4]. The mortality rate in SOT patients reported in the UK and Spanish studies was $11.5 \%$ and $10 \%$, respectively $[9,11]$.

It is assumed that active TB posttransplant develops from the reactivation of old foci of infection in most cases. Nonetheless, studies have reported that only $20 \%-25 \%$ of active TB cases posttransplant were TST-positive before transplantation. The lack of a normal immune response in SOT donors and recipients may be a contributory factor [3]. Transmission of MTB from donated organs has been reported $[12,13]$, and few case reports of nosocomial acquisition of MTB have also been published [14,15].

Several risk factors have been identified in the development of active TB after transplantation. MTB is endemic in several countries, and people born there are at a higher risk of developing TB than those in other countries. Other risk factors include poor environment, smoking, malnutrition, presence of diabetes, chronic kidney disease, and contact with TB patients $[3,16]$. In transplant recipients, the risk of developing posttransplant TB increases with the duration of pre-transplant hemodialysis [17]. In addition, older age and hepatitis C-positive patients are at high risk [16]. Gupta et al. [9] noted that cytomegalovirus positivity was an independent risk factor for posttransplant TB in renal transplant recipients.

\section{CLINICAL MANIFESTATIONS}

Classic symptoms of TB, such as fever, night sweats, and weight loss, may not always be present in transplant recipients. Fever was reported in $91 \%$ of transplant recipients with disseminated disease and in $64 \%$ of patients with pulmonary TB [4]. Moreover, extrapulmonary TB such as meningitis, spondylitis, genitourinary $T B$, intestinal $T B$, and tubercular lymphadenitis have been reported in SOT patients. Rare presentations such as arthritis, tenosynovitis, cutaneous ulcers, and pyomyositis have also been reported $[16,18,19]$.

\section{LABORATORY DIAGNOSIS}

LTBI cases do not show any symptoms or signs of active $T B$, and there will be no radiographic evidence of active TB. TST and IGRAs are two very important tests that can diagnose LTBI cases. TST is considered positive if the skin induration is $\geq 5 \mathrm{~mm}$ in immunosuppressed patients [11]. IGRAs are performed using two methods: QuantiFERONTB Gold (Cellestis, Carnegie, Australia) and T-SPOT test (Oxford Immunotec, Abingdon, UK). Omitted, IGRAs seem to be more specific and sensitive than TST for diagnosing latent TB [20-22]. However, it has been emphasized that 
none of the above tests can reliably differentiate between LTBI and active TB infection cases, and both tests might be falsely negative in immunosuppressed patients $[23,24]$.

The diagnosis of active TB in SOT recipients is challenging. Radiological findings of pulmonary TB in SOT patients may demonstrate focal or diffuse interstitial infiltrates, nodules, pleural effusion, cavitary lesions, or a miliary pattern. In pulmonary TB, samples need to be collected from sputum, gastric lavage, bronchoscopy with bronchoalveolar lavage, or even lung biopsy. In cases of extrapulmonary TB, collection of samples from the site of involvement such as biopsy of the lymph node, pleura, liver, or abscess fluid in patients with soft tissue lesions is recommended. Smear for acid-fast Bacilli, mycobacterial culture, and histopathological evaluation should be performed on the sample [3]. Rapid nucleic acid amplification techniques such as Xpert MTB/resistance to rifampicin (RIF), which is a rapid and sensitive molecular test for MTB and RIF, may be used for the diagnosis of active MTB. However, in cases where few bacteria are present, the test can be falsely negative $[25,26]$.

\section{ASSESSMENT OF TRANSPLANT CANDIDATES AND DONORS}

Evaluation of LTBI is inevitable in all transplant candidates, including those with a history of Bacillus Calmette-Guérin vaccination (BCG). In endemic countries where MTB is prevalent in the community, a thorough history should be taken from all transplant candidates, their donors, and other family members. Any history of active TB should be also noted before transplantation. Chest radiography is an important diagnostic tool for identifying past TB marks $[25,27]$.

TST remains the most commonly used test to detect LTBI. TST is considered positive if there are $\geq 5 \mathrm{~mm}$ indurations at $48-72$ hours. Patients with a negative reaction should have a second TST after 2 weeks to look for any boosting effect. Regardless of TST positivity, all transplant candidates should undergo the IGRA test and have the results interpreted according to the manufacturer's guidelines [28]. Kim et al. [29] demonstrated that the sensitivity of the T-SPOT TB test may be higher than that of TST and suggested that in TST-negative transplant candidates, the T-SPOT TB test might be useful for predicting the risk of developing active TB posttransplant.
In endemic countries with high prevalence of LTBI or asymptomatic active TB, transplant candidates should be evaluated by performing chest radiography and chest computed tomography if feasible, where both TST and IGRA are negative or ambiguous [2,30].

In living organ donation, donors should undergo the same evaluation process as SOT candidates. In deceased organ donation, it is not feasible to perform either TST or IGRA. Hence, a detailed history should be taken from the donor's family regarding previous active TB and any treatment received. If evidence of active TB disease is found or any firm doubt of active TB remains, organs from that donor should not be used for donation $[11,25]$.

\section{TREATMENT OF LATENT TUBERCULOSIS}

The American Thoracic Society, Centers for Disease Control and Prevention, and Infectious Diseases Society of America have suggested LTBI treatment in immunocompromised patients [31]. Holty et al. [32] noted that TB reactivation was significantly less in liver transplant recipients who received LTBI treatment. Hence, they suggested administering treatment to liver transplant recipients with LTBI. Other studies have also suggested LTBI treatment in transplant candidates [33-35].

Isoniazid is the most studied drug for LTBI treatment. Some recommendations have been made for LTBI treatment in transplant recipients $[11,16,25,31,33]$. All transplant candidates and recipients with a positive TST or IGRA should be advised to undergo prophylactic isoniazid treatment. However, patients who have completed an appropriate course of antitubercular therapy for active TB or latent TB may be excluded. Isoniazid can be administered daily or twice weekly by directly observed therapy. Isoniazid therapy can cause neurotoxicity; hence, pyridoxine therapy should be administered along with isoniazid. A 9-month course of isoniazid therapy is preferable to a 6-month course because of the better outcome observed with the former. Even in endemic areas with high TB prevalence, experts have suggested giving ionized prophylaxis to transplant recipients and continuing for 1 year after transplantation [36].

A randomized controlled trial compared a 4-month course of rifampicin monotherapy with a 9-month course of isoniazid monotherapy in the general population and concluded that rifampicin use was associated with a bet- 
ter completion rate, fewer adverse effects, and lower cost [37]. However, rifampicin use can cause significant drug interactions with calcineurin inhibitors (CNIs) in transplant recipients, which may result in graft rejection. Hence, it has been suggested that rifampicin should be completed before transplantation [38].

Another regimen that includes the administration of rifampicin and pyrazinamide daily for 2 months has been evaluated in HIV-infected individuals and found to be effective and less toxic [39-41]. However, later reports of severe hepatotoxicity with rifampicin and pyrazinamide combination made this regimen unfavorable [42].

Sterling et al. [43] demonstrated that a 3-month course of weekly rifapentine plus isoniazid was effective for the treatment of LTBI in the general population. In the case of SOT candidates, the use of this regimen was found to be safe and effective in few case reports [44,45]. However, further large-scale studies are necessary to confirm the safety and efficacy of this regimen.

Isoniazid is generally well tolerated in transplant recipients $[36,46,47]$. In addition, drug interactions between isoniazid and $\mathrm{CNIs}$ have been reported to be insignificant [48]. However, isoniazid-induced hepatotoxicity remains a major concern for clinicians. Guidelines have suggested monitoring liver transaminases in transplant recipients receiving isoniazid. For the first 6 weeks, patients should be monitored closely at 2 weeks interval, and monthly monitoring is adequate afterwards. During the first months of isoniazid therapy, liver enzymes may increase up to 1.5-3 times than normal. This can occur frequently and does not always warrant suspension of the drug administration. However, if there is a threefold rise in hepatic transaminases and the patient has symptoms or signs of hepatotoxicity, or if there is a fivefold increase in hepatic transaminases with or without symptoms, treatment for LTBI should be discontinued $[38,49]$. In liver transplant recipients, the risk of hepatotoxicity may be higher with isoniazid treatment. Some studies have found that the rate of TB reactivation is less than the risk of hepatotoxicity and hence did not suggest giving isoniazid to liver transplant recipients [50-52]. In contrast, other studies demonstrated that isoniazid chemoprophylaxis was well tolerated and therefore suggested to offer treatment to liver transplant recipients who are at high risk of developing TB $[53,54]$.

Fluoroquinolones are emerging as an alternative treatment for patients with LTBI who develop severe isoniazid toxicity. The consensus statement of the group for the study of infection in transplant recipients (GESITRA) rec- ommended the use of ethambutol plus either levofloxacin or moxifloxacin in those patients for at least 6 months [11]. A recent study in 2019 also supported the use of fluoroquinolones for treatment of LTBI in liver transplant candidates and recipients [55].

Studies have reported that most transplant recipients who suffer from active TB after transplantation were TST-negative before transplantation, and only $20 \%-25 \%$ were TST-positive before transplantation [3]. This phenomenon is thought to be due to the high level of anergy to TST in patients with end-stage renal disease and in immunosuppressed transplant recipients [56]. Therefore, experts have recommended the use of prophylactic isoniazid therapy in TST-negative or IGRA-negative patients who have the following: (1) history of untreated TB, (2) history of close and prolonged contact with an active TB case, (3) received an organ from a TST-positive donor, (4) radiological evidence of active TB, or (5) radiological evidence of untreated TB $[11,25,33,38]$.

\section{TREATMENT OF ACTIVE TUBERCULOSIS}

In the general population, guidelines recommended a 6 -month course of treatment for active TB infection. The first 2 months, also called the intensive phase, includes four drugs: isoniazid, rifampicin, pyrazinamide, and ethambutol. The next 4 months, also called the continuation phase, includes two drugs: isoniazid and Rifampicin. If the organism is fully susceptible to isoniazid and Ripampicin in drug susceptibility testing, ethambutol can be safely discontinued before completing the first 2 months. Along with isoniazid, pyridoxine (vitamin B6) should be administered to all patients who are at risk of developing neuropathy. Although different administration schedules are practiced, administration of the drugs once daily during both the intensive and continuation phases is preferred $[25,57]$.

In the case of organ transplant recipients, the most appropriate type and length of therapy remains controversial. Decisions on this are based on case series, general population guidelines, and expert recommendations [26]. Some guidelines recommend the standard 6-month regimen with rifampicin in treating active TB infection [58]. However, other experts have suggested a prolonged course of treatment for at least 9 months in immunosuppressed transplant recipients, as studies have reported that short treatment was associated with a higher risk of relapse 
and death $[59,60]$. The duration of treatment in organ transplants also varies according to the organ involved. For example, 6-9 months of therapy is recommended for bone and joint diseases, 9-12 months in cases of central nervous system involvement, and 6-9 months in cases of disseminated disease. Patients with cavitary pulmonary lesions, whose sputum culture is positive at the end of the first 2 months of the intensive phase or whose response to treatment is slow, should also be given extended treatment for 9 months. In patients who are resistant to rifampicin or other first-line drugs, or who require second-line drugs, the treatment duration should be prolonged. If extended treatment is required, the duration of the continuation phase, rather than the intensive phase, should be prolonged [25]. Other experts have recommended 12-18 months of treatment for extrapulmonary TB and cavitary pulmonary TB with culture positivity after 2 months of therapy [11].

To determine the dosing frequency of antitubercular therapy in transplant patients, daily therapy is preferred over twice or thrice weekly. Studies have reported that intermittent therapy is associated with a higher risk of relapse. In addition, concerns remain that immunosuppressive drug levels may highly fluctuate due to drug-drug interactions with CNIs, which may impact graft outcome [61].

The main problem in using rifampicin-based antitubercular therapy is drug interaction with CNIs. Rifampin is a potent inducer of the cytochrome P450 enzyme, which increases the metabolism of many drugs, including cyclosporine, tacrolimus, sirolimus, everolimus, and corticosteroids to a certain degree. Therefore, the use of rifampicin may result in lower immunosuppressant drug levels, leading to acute graft rejection. Nonetheless, if a rifampicin-based regimen is used, the doses of CNIs and mammalian target of rapamycin (mTOR) inhibitors will have to be increased by at least $2-5$ fold, and the dose of corticosteroid will need to be doubled [62-67].

In some countries, rifampicin-sparing treatment protocols are used for posttransplant TB $[68,69]$. Many physicians prefer not to use rifampicin in transplant recipients because of the high risk of allograft failure. It has been suggested that antitubercular therapy without rifampicin should be continued for an extended period of at least 1218 months in SOT recipients [4]. Bumbacea et al. [60] also suggested a rifampicin-free regimen with isoniazid, pyrazinamide, and Ethambutol for the first 2 months, followed by isoniazid and Ethambutol for a total of 18 months. Another study from India suggested the use of isoniazid, pyrazinamide, and ethambutol for 18 months if rifampicin could not be administered [8].

Fluroquinolones are considered safer options for SOT recipients. Using fluoroquinolones in place of rifampicin, it is easy to skip the hazardous drug interaction of rifampicin [70]. Other studies also found good outcomes with fluoroquinolones [71], and Blumberg et al. [72] suggested that fluoroquinolones may be used as first-line agents. The GESITRA recommended that if rifampicin-free regimens are administered, levofloxacin or moxifloxacin may be added to the maintenance drugs isoniazid and pyrazinamide or ethambutol to reduce the duration of the treatment [11].

Contrary to the above, two clinical trials, the RIFAQUIN trial, in which moxifloxacin was used instead of isoniazid [73], and the OFLOTUB trial, in which ethambutol was replaced with gatifloxacin, demonstrated that the outcome with fluoroquinolones after 4 months of therapy was inferior to that of the standard regimen [74]. Other experts do not recommend moxifloxacin and levofloxacin as first-line therapy; rather, they suggested the use of these drugs in multidrug-resistant (MDR) cases, in patients who developed hepatoxicity with standard therapy, or those with advanced liver disease [25].

However, fluoroquinolone use for a long period of time may result in adverse effects such as arthralgia [3], increased $\mathrm{CNI}$ level [75], and gastrointestinal disturbances [76].

Linezolid has also attracted attention for the treatment of TB. Studies have demonstrated that linezolid is effective against MDR-TB infections. However, patients may develop thrombocytopenia, anemia, and peripheral neuropathy with prolonged use of linezolid [77].

\section{DRUG-RESISTANT MTB}

MDR-TB is caused by MTB, which is resistant to isoniazid and rifampicin. Reports of MDR-TB cases in transplant patients have been published, although the number is small [78-82]. MDR-TB in SOT recipients is associated with extrapulmonary manifestations, severe treatment complications, and death [82]. Chan et al. [83] analyzed the outcome of 205 non-SOT patients with MDR-TB infection treated with second-line anti-TB therapy for 18-24 months and found that the long-term success rate was $75 \%$.

New treatment regimens for MDR-TB have been found to be effective. A prospective multicountry study, involving 
17 countries, analyzed 1,109 patients with MDR-TB who received a multidrug treatment containing bedaquiline, Delamanid, or both for 18 months or more. This study demonstrated that the use of the new regimen resulted in a favorable outcome in $85 \%$ of patients (i.e., conversion of sputum from positive to negative) within 6 months. In contrast, the historical standard of care still in use in much of the world has a $60 \%$ treatment efficacy globally. The authors suggested that these drugs should be urgently made available and accessible to patients with MDR-TB [84]. However, Babar et al. [81] reported severe drug toxicity with the use of a new drug regimen and concluded that the management of MDR-TB in transplant recipients is challenging; hence, close monitoring and follow-up is needed.

\section{CONCLUSION}

MTB remains a challenging opportunistic infection with high morbidity and mortality in SOT recipients. A diagnosis of active TB is very challenging in SOT patients and can only be confirmed by culturing MTB or by identifying specific nucleic acid sequences in a specimen collected from the suspected site. As the development of active TB posttransplant could be life threatening, every effort should be made to prevent active TB via recognition and treatment of LTBI in potential donor and transplant candidates. Current tests for LTBI with TST and IGRA can be falsely negative in patients who are on immunosuppressive medications. Thus, there is a need for better diagnostics for LTBI in SOT recipients. Studies are also needed to investigate the efficacy of newer and shorter treatment regimens, along with their interactions with immunosuppressive medications, and their significant adverse effects. MDR-TB is emerging as a new threat to SOT recipients. New treatment regimens for MDR-TB need to be evaluated for efficacy and safety in transplant patients, and these drugs need to be made available in countries with a high prevalence of MDR-TB.

\section{ACKNOWLEDGMENTS}

\section{Conflict of Interest}

No potential conflict of interest relevant to this article was reported.

\section{ORCID}

Harun Ur Rashid https://orcid.org/0000-0002-9774-2363 Nura Afza Salma Begum

Tasnuva Sarah Kashem

$$
\text { https://orcid.org/0000-0002-5732-0043 }
$$

https://orcid.org/0000-0002-3431-9613

\section{Author Contributions}

Conceptualization: HUR. Visualization: all authors. Writing-original draft: HUR, NASB. Writing-review \& editing: all authors.

\section{Additional Contributions}

We are grateful to Dr. Fowzia Hossain Tanni and Dr. Shanta Rahman for helping with this article. Both of them are Research Assistants at Kidney Foundation Hospital and Research Institute, Bangladesh.

\section{REFERENCES}

1. World Health Organization. Tuberculosis (fact sheets) [Internet]. Geneva: World Health Organization; 2021 [cited 2021 Dec 21]. Available from: https://www.who. int/news-room/fact-sheets/detail/tuberculosis.

2. Currie AC, Knight SR, Morris PJ. Tuberculosis in renal transplant recipients: the evidence for prophylaxis. Transplantation 2010;90:695-704.

3. Muñoz P, Rodríguez C, Bouza E. Mycobacterium tuberculosis infection in recipients of solid organ transplants. Clin Infect Dis 2005;40:581-7.

4. Singh N, Paterson DL. Mycobacterium tuberculosis infection in solid-organ transplant recipients: impact and implications for management. Clin Infect Dis 1998;27:1266-77.

5. Centers for Disease Control and Prevention. World TB day [Internet]. Atlanta, GA; Centers for Disease Control and Prevention; 2016 [cited 2021 Dec 21]. Available from: https://www.cdc.gov/tb/worldtbday/history.htm.

6. World Health Organization. Global tuberculosis report 2021. Geneva: World Health Organization; 2021 [cited 2021 Dec 21]. Available from: https://www.who.int/ publications/i/item/9789240037021.

7. Aubert $O$, Yoo D, Zielinski D, Cozzi E, Cardillo $M$, Dürr $M$, et al. COVID-19 pandemic and worldwide organ transplantation: a population-based study. Lancet Public Health 2021;6:e709-19. 
8. Sakhuja V, Jha V, Varma PP, Joshi K, Chugh KS. The high incidence of tuberculosis among renal transplant recipients in India. Transplantation 1996;61:211-5.

9. Gupta RK, Rosenberg G, Eriksen J, Lipman M, Jackson C, Roy A, et al. Tuberculosis following renal transplantation in England, Wales and Northern Ireland: a national registry-based cohort study. Eur Respir J 2019;54:1802245.

10. Lopez de Castilla D, Schluger NW. Tuberculosis following solid organ transplantation. Transpl Infect Dis 2010;12:106-12.

11. Aguado JM, Torre-Cisneros J, Fortún J, Benito N, Meije $Y$, Doblas A, et al. Tuberculosis in solid-organ transplant recipients: consensus statement of the group for the study of infection in transplant recipients (GESITRA) of the Spanish Society of Infectious Diseases and Clinical Microbiology. Clin Infect Dis 2009;48:1276-84.

12. Centers for Disease Control and Prevention (CDC). Transplantation-transmitted tuberculosis: Oklahoma and Texas, 2007. MMWR Morb Mortal Wkly Rep 2008;57:333-6.

13. Ison MG, Hager J, Blumberg E, Burdick J, Carney K, Cutler $\mathrm{J}$, et al. Donor-derived disease transmission events in the United States: data reviewed by the OPTN/UNOS Disease Transmission Advisory Committee. Am J Transplant 2009;9:1929-35.

14. Sundberg R, Shapiro R, Darras F, Jensen C, Scantlebury $\mathrm{V}$, Jordan $\mathrm{M}$, et al. A tuberculosis outbreak in a renal transplant program. Transplant Proc 1991;23:3091-2.

15. Jereb JA, Burwen DR, Dooley SW, Haas WH, Crawford JT, Geiter LJ, et al. Nosocomial outbreak of tuberculosis in a renal transplant unit: application of a new technique for restriction fragment length polymorphism analysis of Mycobacterium tuberculosis isolates. $\mathrm{J}$ Infect Dis 1993;168:1219-24.

16. Torre-Cisneros J, Doblas A, Aguado JM, San Juan R, Blanes $\mathrm{M}$, Montejo $\mathrm{M}$, et al. Tuberculosis after solid-organ transplant: incidence, risk factors, and clinical characteristics in the RESITRA (Spanish Network of Infection in Transplantation) cohort. Clin Infect Dis 2009;48:1657-65.

17. Torres J, Aguado JM, San Juan R, Andrés A, Sierra P, López-Medrano F, et al. Hepatitis $C$ virus, an important risk factor for tuberculosis in immunocompromised: experience with kidney transplantation. Transpl Int 2008;21:873-8.

18. Murugesh Anand S, Edwin Fernando M, Srinivasaprasad ND, Sujit S, Thirumalvalavan K. Tuberculous myo- sitis and cellulitis in a renal transplant recipient. Indian J Tuberc 2020;67:353-6.

19. Toyokawa N, Kokubu T, Fujioka H. Mycobacterial tuberculous tenosynovitis of the extensor tendon occurring after liver transplantation: a case report. Hand Surg 2008;13:37-40.

20. Kim SY, Jung GS, Kim SK, Chang J, Kim MS, Kim YS, et al. Comparison of the tuberculin skin test and interferon- $\gamma$ release assay for the diagnosis of latent tuberculosis infection before kidney transplantation. Infection 2013;41:103-10.

21. Mazurek GH, Jereb J, Vernon A, LoBue P, Goldberg S, Castro $\mathrm{K}$, et al. Updated guidelines for using Interferon gamma release assays to detect Mycobacterium tuberculosis infection: United States, 2010. MMWR Recomm Rep 2010;59:1-25.

22. Mazurek GH, LoBue PA, Daley CL, Bernardo J, Lardizabal AA, Bishai WR, et al. Comparison of a whole-blood interferon gamma assay with tuberculin skin testing for detecting latent Mycobacterium tuberculosis infection. JAMA 2001;286:1740-7.

23. Sester $U$, Wilkens $H$, van Bentum $K$, Singh $M$, Sybrecht GW, Schäfers HJ, et al. Impaired detection of Mycobacterium tuberculosis immunity in patients using high levels of immunosuppressive drugs. Eur Respir $\mathrm{J}$ 2009;34:702-10.

24. Lange B, Vavra M, Kern WV, Wagner D. Indeterminate results of a tuberculosis-specific interferon-gamma release assay in immunocompromised patients. Eur Respir J 2010;35:1179-82.

25. Subramanian A, Dorman S; AST Infectious Diseases Community of Practice. Mycobacterium tuberculosis in solid organ transplant recipients. Am J Transplant 2009;9 Suppl 4:S57-62.

26. Meije $Y$, Piersimoni C, Torre-Cisneros J, Dilektasli AG, Aguado JM; ESCMID Study Group of Infection in Compromised Hosts. Mycobacterial infections in solid organ transplant recipients. Clin Microbiol Infect 2014;20 Suppl 7:89-101.

27. Rubin $\mathrm{RH}$. Infection in the organ transplant recipient. In: Rubin RH, Young LS, Van Furth R, eds. Clinical approach to infection in the compromised host. 4th ed. Boston, MA: Springer; 2002. p. 573-679.

28. National Collaborating Centre for Chronic Conditions (UK); Centre for Clinical Practice at NICE (UK). Tuberculosis: clinical diagnosis and management of tuberculosis, and measures for its prevention and control. NICE clinical guidelines, No. 117. The guideline: diagnosis 
and treatment. London: National Institute for Health and Clinical Excellence (UK); 2011 [cited 2021 Dec 21]. Available from: https://www.ncbi.nlm.nih.gov/books/ NBK97854/.

29. Kim SH, Lee SO, Park IA, Park SJ, Choi SH, Kim YS, et al. Diagnostic usefulness of a $T$ cell-based assay for latent tuberculosis infection in kidney transplant candidates before transplantation. Transpl Infect Dis 2010;12:113-9.

30. Lyu J, Lee SG, Hwang S, Lee SO, Cho OH, Chae EJ, et al. Chest computed tomography is more likely to show latent tuberculosis foci than simple chest radiography in liver transplant candidates. Liver Transpl 2011;17:9638.

31. American Thoracic Society; Centers for Disease Control and Prevention; Infectious Diseases Society of America. American Thoracic Society/Centers for Disease Control and Prevention/Infectious Diseases Society of America: controlling tuberculosis in the United States. Am J Respir Crit Care Med 2005;172:1169-227.

32. Holty JE, Gould MK, Meinke L, Keeffe EB, Ruoss SJ. Tuberculosis in liver transplant recipients: a systematic review and meta-analysis of individual patient data. Liver Transpl 2009;15:894-906.

33. Blumberg HM, Leonard MK Jr, Jasmer RM. Update on the treatment of tuberculosis and latent tuberculosis infection. JAMA 2005;293:2776-84.

34. Menzies D, Al Jahdali H, Al Otaibi B. Recent developments in treatment of latent tuberculosis infection. Indian J Med Res 2011;133:257-66.

35. Jafri SM, Singal AG, Kaul D, Fontana RJ. Detection and management of latent tuberculosis in liver transplant patients. Liver Transpl 2011;17:306-14.

36. Naqvi R, Akhtar S, Noor H, Saeed T, Bhatti S, Sheikh R, et al. Efficacy of isoniazid prophylaxis in renal allograft recipients. Transplant Proc 2006;38:2057-8.

37. Aspler A, Long R, Trajman A, Dion MJ, Khan K, Schwartzman $\mathrm{K}$, et al. Impact of treatment completion, intolerance and adverse events on health system costs in a randomised trial of 4 months rifampin or 9 months isoniazid for latent TB. Thorax 2010;65:582-7.

38. Targeted tuberculin testing and treatment of latent tuberculosis infection. American Thoracic Society. MMWR Recomm Rep 2000;49:1-51.

39. Halsey NA, Coberly JS, Desormeaux J, Losikoff P, Atkinson J, Moulton LH, et al. Randomised trial of isoniazid versus rifampicin and pyrazinamide for prevention of tuberculosis in HIV-1 infection. Lancet 1998;351:786-
92.

40. Mwinga A, Hosp M, Godfrey-Faussett $P$, Quigley $M$, Mwaba P, Mugala BN, et al. Twice weekly tuberculosis preventive therapy in HIV infection in Zambia. AIDS 1998;12:2447-57.

41. Gordin F, Chaisson RE, Matts JP, Miller C, de Lourdes Garcia M, Hafner R, et al. Rifampin and pyrazinamide vs isoniazid for prevention of tuberculosis in HIV-infected persons: an international randomized trial. Terry Beirn Community Programs for Clinical Research on AIDS, the Adult AIDS Clinical Trials Group, the Pan American Health Organization, and the Centers for Disease Control and Prevention Study Group. JAMA 2000;283:1445-50.

42. Jasmer RM, Daley CL. Rifampin and pyrazinamide for treatment of latent tuberculosis infection: is it safe? Am J Respir Crit Care Med 2003;167:809-10.

43. Sterling TR, Villarino ME, Borisov AS, Shang N, Gordin F, Bliven-Sizemore E, et al. Three months of rifapentine and isoniazid for latent tuberculosis infection. $\mathrm{N}$ Engl $\mathrm{J}$ Med 2011;365:2155-66.

44. de Castilla DL, Rakita RM, Spitters CE, Narita M, Jain $\mathrm{R}$, Limaye AP. Short-course isoniazid plus rifapentine directly observed therapy for latent tuberculosis in solid-organ transplant candidates. Transplantation 2014;97:206-11.

45. Knoll BM, Nog R, Wu Y, Dhand A. Three months of weekly rifapentine plus isoniazid for latent tuberculosis treatment in solid organ transplant candidates. Infection 2017;45:335-9.

46. Lui SL, Li FK, Choy BY, Chan TM, Lo WK, Lai KN. Longterm outcome of isoniazid prophylaxis against tuberculosis in Chinese renal transplant recipients. Transpl Infect Dis 2004;6:55-6.

47. Dodani SK, Nasim A, Aziz T, Naqvi A. The efficacy of isoniazid prophylaxis in renal transplant recipients in a high tuberculosis burden country. Transpl Infect Dis 2021;23:e13709.

48. Sud K, Muthukumar T, Singh B, Garg SK, Kohli HS, Jha $V$, et al. Isoniazid does not affect bioavailability of cyclosporine in renal transplant recipients. Methods Find Exp Clin Pharmacol 2000;22:647-9.

49. Yee D, Valiquette C, Pelletier M, Parisien I, Rocher I, Menzies D. Incidence of serious side effects from firstline antituberculosis drugs among patients treated for active tuberculosis. Am J Respir Crit Care Med 2003;167:1472-7.

50. Nagai S, Fujimoto Y, Taira K, Egawa H, Takada Y, Kiuchi 
T, et al. Liver transplantation without isoniazid prophylaxis for recipients with a history of tuberculosis. Clin Transplant 2007;21:229-34.

51. Benito N, Sued O, Moreno A, Horcajada JP, González J, Navasa $M$, et al. Diagnosis and treatment of latent tuberculosis infection in liver transplant recipients in an endemic area. Transplantation 2002;74:1381-6.

52. Fábrega E, Sampedro B, Cabezas J, Casafont F, Mieses MÁ, Moraleja I, et al. Chemoprophylaxis with isoniazid in liver transplant recipients. Liver Transpl 2012;18:1110-7.

53. Singh N, Wagener MM, Gayowski T. Safety and efficacy of isoniazid chemoprophylaxis administered during liver transplant candidacy for the prevention of posttransplant tuberculosis. Transplantation 2002;74:8925.

54. Stucchi RS, Boin IF, Angerami RN, Zanaga L, Ataide EC, Udo EY. Is isoniazid safe for liver transplant candidates with latent tuberculosis? Transplant Proc 2012;44:2406-10.

55. Silva JT, San-Juan R, Fernández-Ruiz M, Aguado JM. Fluoroquinolones for the treatment of latent Mycobacterium tuberculosis infection in liver transplantation. World J Gastroenterol 2019;25:3291-8.

56. Shankar MS, Aravindan AN, Sohal PM, Kohli HS, Sud K, Gupta KL, et al. The prevalence of tuberculin sensitivity and anergy in chronic renal failure in an endemic area: tuberculin test and the risk of post-transplant tuberculosis. Nephrol Dial Transplant 2005;20:2720-4.

57. Nahid P, Dorman SE, Alipanah N, Barry PM, Brozek $\mathrm{JL}$, Cattamanchi A, et al. Official American Thoracic Society/Centers for Disease Control and Prevention/Infectious Diseases Society of America clinical practice guidelines: treatment of drug-susceptible tuberculosis. Clin Infect Dis 2016;63:e147-95.

58. EBPG Expert Group on Renal Transplantation. European best practice guidelines for renal transplantation. Section IV: long-term management of the transplant recipient. IV.7.2. Late infections. Tuberculosis. Nephrol Dial Transplant 2002;17 Suppl 4:39-43.

59. al-Sulaiman $\mathrm{MH}$, Dhar JM, al-Khader AA. Successful use of rifampicin in the treatment of tuberculosis in renal transplant patients immunosuppressed with cyclosporine. Transplantation 1990;50:597-8.

60. Bumbacea D, Arend SM, Eyuboglu F, Fishman JA, Goletti $D$, Ison $M G$, et al. The risk of tuberculosis in transplant candidates and recipients: a TBNET consensus statement. Eur Respir J 2012;40:990-1013.
61. Chang KC, Leung CC, Yew WW, Chan SL, Tam CM. Dosing schedules of 6-month regimens and relapse for pulmonary tuberculosis. Am J Respir Crit Care Med 2006;174:1153-8.

62. Buffington GA, Dominguez JH, Piering WF, Hebert LA, Kauffman HM Jr, Lemann J Jr. Interaction of rifampin and glucocorticoids: adverse effect on renal allograft function. JAMA 1976;236:1958-60.

63. Paterson DL, Singh N. Interactions between tacrolimus and antimicrobial agents. Clin Infect Dis 1997;25:143040.

64. McAllister WA, Thompson PJ, Al-Habet SM, Rogers HJ. Rifampicin reduces effectiveness and bioavailability of prednisolone. Br Med J (Clin Res Ed) 1983;286:923-5.

65. Modry DL, Stinson EB, Oyer PE, Jamieson SW, Baldwin JC, Shumway NE. Acute rejection and massive cyclosporine requirements in heart transplant recipients treated with rifampin. Transplantation 1985;39:313-4.

66. Ngo BT, Pascoe M, Khan D. Drug interaction between rifampicin and sirolimus in transplant patients. Saudi J Kidney Dis Transpl 2011;22:112-5.

67. Lefeuvre S, Rebaudet S, Billaud EM, Wyplosz B. Management of rifamycins- everolimus drug-drug interactions in a liver-transplant patient with pulmonary tuberculosis. Transpl Int 2012;25:e120-3.

68. Vachharajani TJ, Oza UG, Phadke AG, Kirpalani AL. Tuberculosis in renal transplant recipients: rifampicin sparing treatment protocol. Int Urol Nephrol 2002;34:551-3.

69. Jha V, Sakhuja V. Rifampicin sparing treatment protocols in posttransplant tuberculosis. Int Urol Nephrol 2004;36:287-8.

70. Ho CC, Chen YC, Hu FC, Yu CJ, Yang PC, Luh KT. Safety of fluoroquinolone use in patients with hepatotoxicity induced by anti-tuberculosis regimens. Clin Infect Dis 2009;48:1526-33.

71. Yoon HE, Jeon YJ, Chung HW, Shin SJ, Hwang HS, Lee SJ, et al. Safety and efficacy of a quinolone-based regimen for treatment of tuberculosis in renal transplant recipients. Transplant Proc 2012;44:730-3.

72. Blumberg HM, Burman WJ, Chaisson RE, Daley CL, Etkind SC, Friedman LN, et al. American Thoracic Society/Centers for Disease Control and Prevention/ Infectious Diseases Society of America: treatment of tuberculosis. Am J Respir Crit Care Med 2003;167:60362.

73. Jindani A, Harrison TS, Nunn AJ, Phillips PP, Churchyard GJ, Charalambous $S$, et al. High-dose rifapentine with moxifloxacin for pulmonary tuberculosis. $\mathrm{N}$ Engl $\mathrm{J}$ 
Med 2014;371:1599-608.

74. Merle CS, Fielding K, Sow OB, Gninafon M, Lo MB, Mthiyane $\mathrm{T}$, et al. A four-month gatifloxacin-containing regimen for treating tuberculosis. $\mathrm{N}$ Engl $\mathrm{J}$ Med 2014;371:1588-98.

75. Kirsch S, Sester M. Tuberculosis in transplantation: diagnosis, prevention, and treatment. Curr Infect Dis Rep 2012;14:650-7.

76. Lou HX, Shullo MA, McKaveney TP. Limited tolerability of levofloxacin and pyrazinamide for multidrug-resistant tuberculosis prophylaxis in a solid organ transplant population. Pharmacotherapy 2002;22:701-4.

77. Fortún J, Martín-Dávila P, Navas E, Pérez-Elías MJ, Cobo J, Tato $\mathrm{M}$, et al. Linezolid for the treatment of multidrug-resistant tuberculosis. J Antimicrob Chemother 2005;56:180-5.

78. Wong CF, Fung SL, Yan SW, Lee J, Cheng LC, Chiu CS. Lung transplantation in Hong Kong: 12 years of experience. Respirology 2008;13:903-7.

79. Di Perri G, Luzzati R, Forni A, Allegranzi B, Cazzadori A, Bonora $S$, et al. Fatal primary multidrug-resistant tuberculosis in a heart transplant recipient. Transpl Int
1998;11:305-7.

80. Shitrit D, Bendayan D, Saute M, Kramer MR. Multidrug resistant tuberculosis following lung transplantation: treatment with pulmonary resection. Thorax 2004;59:79-80.

81. Babar ZU, Nasim A, Kumar S, Nazmi J, Badlani S, Na$\operatorname{deem} A$, et al. A case series of multidrug-resistant tuberculosis in renal transplant recipients: challenges in management from a TB endemic country. Transpl Infect Dis 2021;23:e13659.

82. Huaman MA, Brawley R, Ashkin D. Multidrug-resistant tuberculosis in transplant recipients: case report and review of the literature. Transpl Infect Dis 2017;19.

83. Chan ED, Laurel V, Strand MJ, Chan JF, Huynh ML, Goble M, et al. Treatment and outcome analysis of 205 patients with multidrug-resistant tuberculosis. Am J Respir Crit Care Med 2004;169:1103-9.

84. Franke MF, Khan $P$, Hewison $C$, Khan $U$, Huerga $H$, Seung KJ, et al. Culture conversion in patients treated with bedaquiline and/or delamanid: a prospective multicountry study. Am J Respir Crit Care Med 2021;203:111-9. 